\section{Applications of isoelectric focusing}

Biological and Biomedical Applications of Isoelectric Focusing. Edited by N. Catsimpoolas and J. Drysdale. (Plenum: New York, 1977.) \$39.

ANY collection of the applications of a specialised technique in science is open to two criticisms: firstly that it inherently only appeals to a small group of readers, as the bulk of the applications will be of little interest to them; and secondly that such collections are liable to be outdated even before publication. The editors of this book have almost avoided these pitfalls, the first by selecting a subject which is of wide application and therefore of general interest in protein chemistry, and the second by choosing a technique the methodology of which has changed little in the past six years. They are thus able virtually to ignore the basic techniques of isoelectric focusing, except in so far as it is introduced by the individual authors.

Chapters 1, 2, 5 and 10 will be of special interest to the clinical chemist, as they deal with the analysis of the body fluids using isoelectric focusing, with special emphasis on the haemoglobins and immunoglobins in chapters 2 and 5 . Chapter 5 is particularly valuable, as the technique of isoelectric focusing has made fundamental contributions to the problems of antibody heterogeneity and to the study of antibody synthesis, both in vivo and in vitro. Hoffman provides an excellent, up-to-date account of this subject. Latner (chapter 10) gives a very complete review of the applications of his two-dimensional isoelectric focusinggel electrophoresis technique to serum proteins in normal and pathological conditions. Although two-dimensional methods are notoriously difficult to reproduce, the complexity of the protein components in serum necessitates this approach, and Latner's illustrations illustrate the value of the method in clinical chemistry.

More specialised chapters are contributed by Florini on muscle proteins and by Schmidt-Ullrich and Wallach on membrane proteins. Both these groups are notorious for solubility and aggregation problems which may necessitate the use of strong urea, and/or detergent solutions, both for extraction and for fractionation. Both reviewers cover these points in detail and offer methods for their solution.

The application of isoelectric focusing in plant biochemistry is well illustrated by Wrigley's chapter 8 on seed proteins. Here again, the number of protein components present in crude extracts has necessitated the development of two-dimensional methods both for species identification and for genetic studies. The lectins, a class of plant proteins that are becoming increasingly important as carbohydratespecific biochemical reagents, are also covered in this review, which makes refreshing reading for the more classically minded biochemist.

I found MacGillivray's review on the use of isoelectric focusing in the separation and characterisation of nuclear non-histone proteins particularly interesting and instructive. It provides an excellent example of the way in which sophisticated analytical electrophoretic methods can be used to monitor and design preparative methods for the isolation of even minor components of complex mixtures. The components themselves are

\section{Astro-art}

The New Challenge of the Stars: A Science Fact Look at Science Fiction. By Patrick Moore and David Hardy. Foreword by Arthur C. Clarke. Pp. 62. (Mitchell Beazley: London, 1978.) $£ 4.95$.

Astronomy and art are frequent bedfellows. The astro-artists, with brush, palette and Allen's Astrophysical Quantities to hand, can easily transcend the barriers of time and space, leaving the astronomer and astrophysicist shackled in the present and to the immediate vicinity of the Sun. He can paint what the eyes of mankind can never see. We can be transported to the birth of the Solar System, to the centre of a globular cluster, to the edge of a supernova explosion. The endproducts are paintings of considerable vibrance, colour and beauty but more than that they are paintings of considerable educative worth. The artist molds his scene to embody all the factual information available at the time. The scientist and scholar, as well as enjoying the paintings, can use them as vivid inages of present-day knowledge, an easily assimilatable set of stepping stones to future theories and hypotheses.

The New Challenge of the Stars is a large format book $(23 \times 33 \mathrm{~cm})$ and contains 30 or so full-page to double-page reproductions of David Hardy's space paintings. Half the paintings are space fact, my favourites being "An unmanned probe re-appears after having passed through the coma of the comet"-the ghostly form of the comet illuminating the Mariner-type spacecraft with the crescent Earth and Moon hovering in the distance; "Saturn as seen from Rhea"-. Rhea assumed to have a Moon-like surface being completely menaced by the leaden domineering planet with its edge often intractable in nature, and available only in small quantities, but they may nevertheless be of key importance in gene expression.

While concluding that this is very often a valuable source book, may I plead for a moriatorium on books on isoelectric focusing. At least one major volume has been devoted to this relatively small field every year since 1973, and the subject matter is getting very well-worn. In any case revolutionary new advances in preparative isoelectric focusing are imminent, and it would be well to wait for these to be incorporated into the literature, before attempting further surveys.

C. J. O. R. Morris

C. J. O. R. Morris is Emeritus Professor of Experimental Biochemistry, formerly at the London Hospital Medical College and Queen Mary College, University of London, UK.

on rings slicing razor sharp across the sky; and "Icarus"- barren and cratered, diffused in the red glow of Mars with the Sun ominously emerging from eclipse.

The remainder of the pictures are from the realms of science fiction and fantasy. We see a crippled spacecraft lying like a wounded snake across the surface of a planet, crawling with robot repair vehicles, the sky behind filled with the splendour of a giant star pouring plasma into a black hole; we have asteroids hollowed out to produce space Arks, space colonies being attacked by alien civilisations, the desolation of futuristic cities after the nova explosion of their stellar suns, lunar and Martian bases, and embryonic space towns orbiting Earth.

Patrick Moore's text is business-like and informative and geared to the inquisitiveness of the amateur astronomer. However, the meat of this book is the artwork. It is magnificent and David Hardy will enthuse and enchant today's young astronomers in exactly the way that the previous generation marvelled at the paintings of Chesley Bonestell in The Conquest of Space (text by Willy Ley; Sidgwick and Jackson: London, 1950) and of R. A. Smith in Exploration of the Moon (text by Arthur C. Clarke; Frederick Muller: London, 1954).

Astro-art has been with us a long time; this book and the ever increasing popularity of the American monthly magazine Astronomy show that it is in an extremely healthy state today. In the future we will realise that some of Hardy's visions are incorrect. But the enjoyment and wonder they engender today in the minds of young readers will have played a great part in motivating them to strive for more knowledge, knowledge which will possibly prove him wrong.

David W. Hughes

David W. Hughes is Lecturer in Physics and Astronomy at the University of Sheffield, UK. 